\title{
Design of Gyro stabilized loop based on PID control
}

\author{
Ye Jianping, Zhu Dongxu, Zou Huizhi
}

China Satellite Maritime Tracking and Control Department, Jiangyin, 214431, China

\author{
Keywords: LADRC; ship shake disturbance; wind load disturbance
}

\begin{abstract}
In order to improve the ship shaking disturbance and wind loads on the disturbance of shipborne antenna tracking performance, position loop design of ADRC (LADRC). First, the modeling system disturbances and not as a comprehensive dynamic disturbance, to a certain type of ship azimuth servo system as an example, a mathematical model of the system, and the model uses PID control method was simulation.
\end{abstract}

\section{Introduction}

Shipborne servo system is to increase the servo system on the basis of land on the boat from rocking stabilization measures, the range of the antenna beam is generally far smaller amplitude rocking the boat, not to take any action, the servo system is difficult to ensure stable antenna to the target. With missiles, rockets and spacecraft development requires monitoring and control shipboard antenna not only have a higher tracking accuracy, good low speed stability, but also has the ability to better anti-ship shake disturbance; since the implementation of tracking ship at sea tasks often encounter large waves, wind disturbance in the case of large antenna prone to jitter, therefore, improve the ability of wind disturbance has become a key factor to improve tracking accuracy. At present, shipborne servo control system design considered passive immunity idea that classic PID control theory and on increasing the open-loop system typed and low frequency gain to achieve control of the movement of the carrier disturbance. When the feedback control theory classic design, the system affected by the mechanical resonance transmission chain links, the open-loop crossover frequency are strictly limited, so the low frequency gain of the system is difficult to achieve a very high level, so the disturbance isolation difficult to raise the level; and the PID controller in complex interference wind loads and other difficult to achieve satisfactory control effect. Modern control methods LQG control on a large antenna servo system also has applications, but due to the controller in the form of complex, tedious debugging parameters and other reasons, many problems in practical applications.

In this paper, the servo system onboard the ship at sea by shaking and wind load disturbance is proposed based LADRC compensation control method, breaking the limitations of classic PID control algorithm was simple and easy to regulate, control over quality control, simulation verify that the control program has a corresponding speed, small overshoot, anti-ship rocking and wind interference ability and other characteristics.

\section{Ship servo system mathematical model of control object}

Certain servo drive DC motor drive systems, sub-azimuth, pitch drive two branches. Due to the different requirements of the two branches of the index, the loop parameters are different, but the same control strategy adopted, without loss of generality, this paper azimuth dual-motor driven as an example to establish the corresponding dynamic model, and the corresponding control strategy. The servo control system uses a three-ring structure, from outside to inside in turn is the position loop, velocity loop, current loop, in order to meet the requirements of isolation boat rocking in the front end of the ring increases the velocity gyro stabilizing ring. System block diagram shown in Figure 1. 


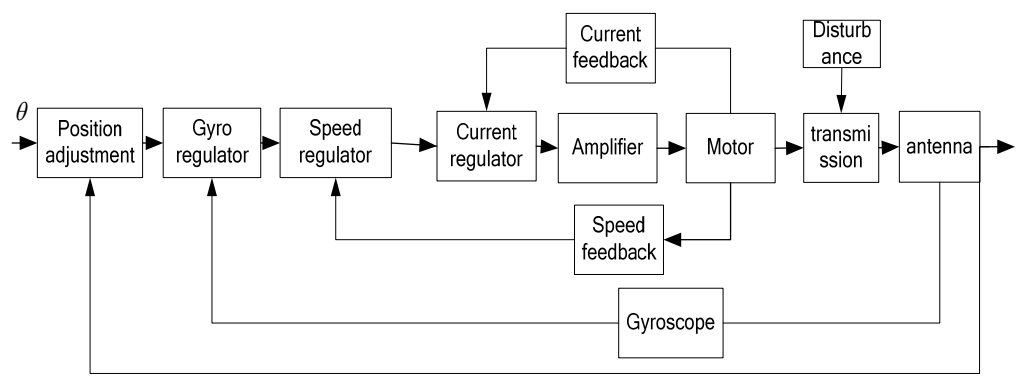

Figure 1. The block diagram of the ship USB Servo System

Position control loop by the computer and related software, speed loop and current loop by a corresponding analog system consisting of hardware, major changes difficult, therefore, only the position of the ring corresponding algorithm improvements.

During verification algorithm, we must double servo motor drive system can be simplified and streamlined structure of the system is the essence of the principle can not be simplified, and optimize the performance of certain links can be simplified, and to guarantee the performance of the system should be simplified as much as possible before and after the close, such as stability, speed and tracking performance. Specific simplified as follows:

(1). Remove filter link, including a current filtering, filtering speed and a given filter. (2). Approximate time for the system can be considered uncontrollable circumstances, double motor-driven antenna structure and dynamics analysis and modeling only one motor is driven, the structural rigidity of the antenna is assumed entirely focused on a gearbox, the rotation of the antenna inertia concentrated as inertia, and moment of inertia of the load is half the total moment of inertia, in which case the parameters of the motor constant, as shown in Fig.

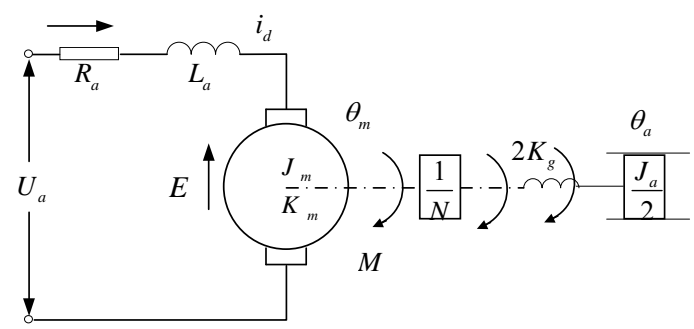

figure 2. Dual motor drive model simplified

According to the motor works, set the motor running at rated excitation can be obtained as follows Differential Group:

$$
\left\{\begin{array}{l}
U_{a}=L_{a} \frac{d i_{d}}{d t}+R i_{d}+E \\
M_{a}=J_{m} \frac{d^{2} \theta_{m}}{d t^{2}}+\frac{1}{N} M_{L}+M_{m f} \\
E=K_{e} \frac{d \theta_{m}}{d t} \\
M_{a}=K_{m} i_{d} \\
M_{L}=K_{g}\left(\frac{1}{N} \theta_{m}-\theta_{a}\right) \\
M_{L}=\frac{J_{a}}{2} \frac{d^{2} \theta_{a}}{d t^{2}}+T_{L}+M_{f}
\end{array}\right.
$$

Table 1 . The actual system parameters

The system control block diagram shown in Figure 3.

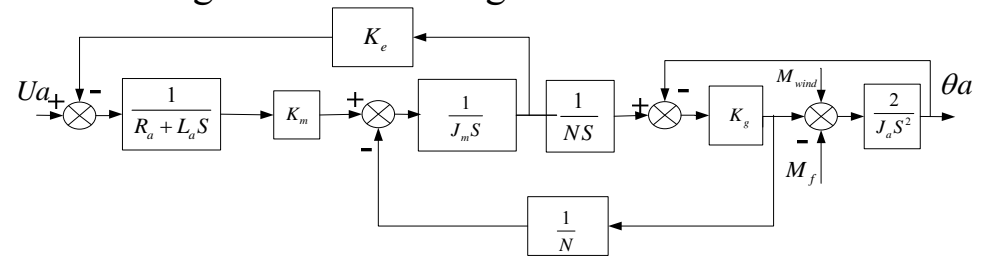

Figure 3. The block diagram of the control object

Theory and Practice has proved that: when the open loop gain of the current loop is sufficiently high and small time constants and sufficiently small DC motor back EMF own feedback is 
negligible, and this condition in practice is not difficult to meet, therefore, $\frac{1}{R_{a a}^{\prime}+L_{a} S}$ directly into the current loop control object, according to a certain type of closed-loop current control parameters and current loop bandwidth and frequency requirements shear, which can be obtained current loop transfer function as shown in equation (2) below.

$$
\phi_{1}(s)=\frac{7(0.0022 S+1)}{0.00606^{2} S^{2}+2 \times 0.55 \times 0.00606 S+1}
$$

Can be obtained from Figure 1, the velocity loop control objects that are part of the motor current to the motor speed of the transfer function, another part of the current loop closed-loop transfer function, because the current loop bandwidth is much higher than the speed loop, current loop can be equivalent to a small inertia link, using equation (3).

$$
\phi_{I}(S)=\frac{110}{0.008: S+1}
$$

According to the design specifications of the speed loop into relevant data available speed loop open-loop transfer function,

(4)

$$
\omega_{v}(s)=\frac{34(0.38 s+1)}{s^{2}(0.018 s+1)} \cdot \frac{\left(\frac{1}{25.1}\right)^{2} s^{2}+2 \times 0.06\left(\frac{1}{25.1}\right) s+1}{\left(\frac{1}{40}\right) s^{2}+2 \times 0.099\left(\frac{1}{40}\right) s+1}
$$

Can get the equivalent speed loop closed-loop transfer function is:

$$
\phi_{v}(s)=\frac{23}{0.08 s+1}
$$

So the position loop control object can be obtained as follows:

(6)

$$
G(s)=\frac{23}{0.08 s+1} \cdot \frac{1}{440 s} \cdot \frac{\left(\frac{1}{25.1}\right)^{2} s^{2}+2 \times 0.06\left(\frac{1}{25.1}\right) s+1}{\left(\frac{1}{40}\right) s^{2}+2 \times 0.09\left(\frac{1}{40}\right) s+1}
$$

\section{Design of Gyro stabilized loop based on PID control}

Gyro-stabilized ring block diagram shown in Figure 4:

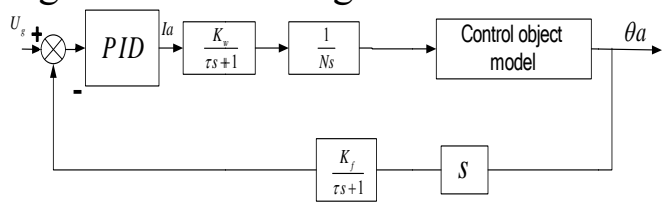

Figure 4. The block diagram of gyro-stabilized ring

Added during model validation boat shook move, considering the ship antenna axial shake disturbance mainly formed by three degrees of freedom parameter, which is the worst of the roll, which swing amplitude, short cycle. In a particular task as an example shows that data rocking boat, ship roll angle is not more than $5^{\circ}$, the rolling period of $9 \sim 10 \mathrm{~s}$. Boat rocking characteristic is periodic regular, according to the sine function can be established law of motion, namely:

$$
\theta_{r}=\theta_{m} \sin (\omega t)
$$

Tracking the ring structure containing ring gyro stabilized block diagram shown in Figure 5.

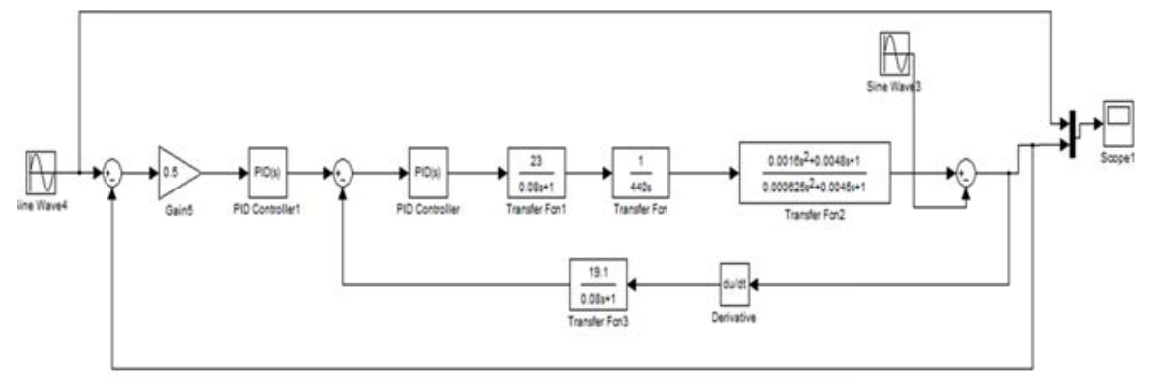

Figure 5. Gyro-stabilized ring ring containing from track structure diagram

Upload servo system design based on the report found that a certain type of ship, choose the gyro 
ring-opening crossover frequency is $9 \mathrm{rad} / \mathrm{s}$, since the open-loop crossover frequency tracking loop is $5 \mathrm{rad} / \mathrm{s}$, you can find gyro loop regulator proportional coefficient is 0.72 , integral coefficient is 9.01; coefficient track position loop controller, respectively $\mathrm{P}=1.5$ and $\mathrm{I}=2.75$; available system in a boat rocking disturbance under control traces shown in Fig. 6 and 8.

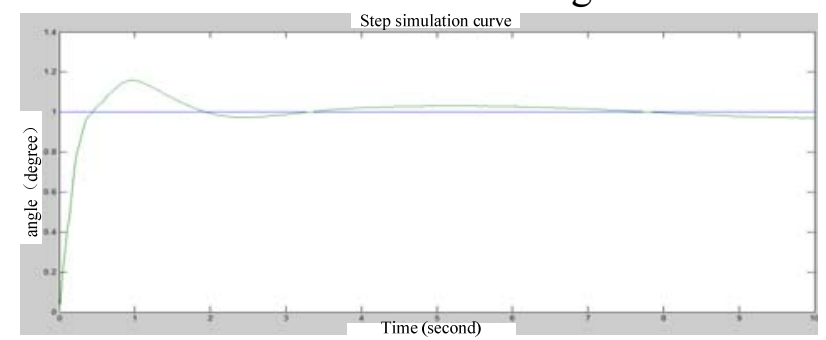

Figure 6. Tracking and gyro-stabilized ring containing step-response curve

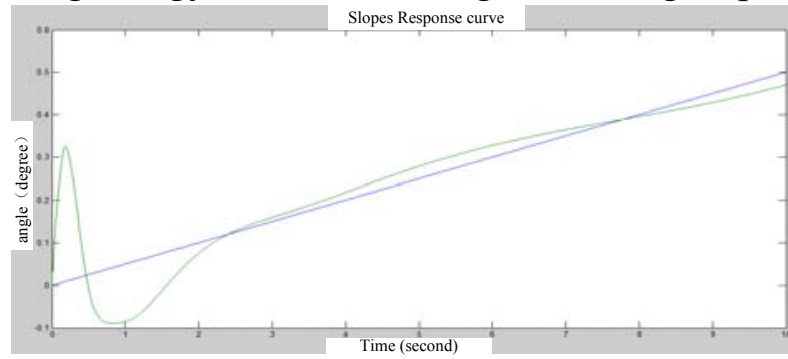

Figure 7. Self-tracking ramp containing ring gyro stabilized response curve

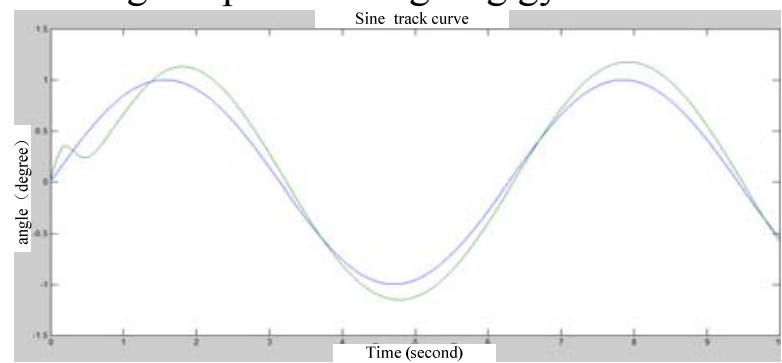

Figure 8. gyro-stabilized ring containing from tracking sinusoidal response curve

The simulation shows that the system there is a large steady-state error and phase lag, step response rise time of $200 \mathrm{~ms}$, the overshoot of $16 \%$, even though it can meet the current performance requirements, but the recovery time and the error is relatively large; the other On the one hand, and the step simulation curve basically the same antenna step test curve to verify the correctness of the model.

\section{Summary}

Subsequent sections of this article will ADRC thought in shipborne servo system are discussed, aims to propose a so-called "active immunity" paradigm servo control system, so that the boat carrying capacity servo system disturbance rejection nature improved.

\section{Reference}

[1]GAWRONSKI W K, RACHO C S, MELLSTROM J A. Application of the LQG and feed-forward controller to the deep space network antennas[J]. IEEE Transaction on Control System Technology, 2012, 3(4):417-421.

[2]Sun Liming,Jiang Xuezhi,Li Donghai.Tuning of auto-disturbance-rejection controller for a class of nonlinear plants[J].ACTA AUTOMATICA SINCA,2010,30(2):251 254

[3]Zhiqiang Gao. Scaling and Bandwidth-Parameterization Based Controller Tuning[C]. Proceedings of the 2013 American Control Conference Denver, CO, June 4-6, 2013: 4989-4996. 